\title{
Herramientas para la evaluación de la disnea y el compromiso respiratorio en pacientes atendidos por teléfono o video-llamada en el contexto de la pandemia por COVID-19
}

Tools for the evaluation of dyspnea and respiratory compromise in patients treated by phone or video-call in the context of COVID-19 pandemic

\section{Comentado de:}

Greenhalgh $T$ y col. 2020, The Center for Evidence-Based Medicine. University of Oxford ${ }^{1}$

\section{Introducción}

En el contexto de la pandemia por COVID-19, y con la finalidad de reducir la posible propagación del virus, los profesionales de la salud de muchas regiones del mundo están minimizando el contacto cara a cara con sus pacientes, utilizando la consulta telefónica o las video-llamadas como primera alternativa de contacto.

Por esta razón, los autores realizaron una revisión no sistemática sobre las principales herramientas disponibles para evaluar en forma remota la disnea en particular y el compromiso respiratorio en general de quien está consultando. Sin embargo, no encontraron evidencia de que la medición a distancia de la frecuencia respiratoria sea precisa en este contexto. Por otro lado, experiencia anecdótica reciente con pacientes con COVID-19 sugiere que el puntaje de Roth no sería lo suficientemente sensible como para identificar personas con enfermedad respiratoria grave y que podría conducir a brindar un falso reaseguro.

Por estas razones, las recomendaciones que ofrecen -una lista de siete preguntas- se basan en opiniones de expertos obtenidas a través de una encuesta rápida realizada el 20/3/2020 a una muestra de 50 médicos británicos que ya venían evaluando regularmente pacientes por teléfono.

\section{Herramientas para evaluar a distancia el compromiso respiratorio}

\section{Evaluación de la frecuencia respiratoria}

Los autores de esta revisión narrativa no encontraron evidencia de que la medición de la frecuencia respiratoria sea precisa cuando es realizada en el contexto de la atención a distancia, sin que los expertos consultados usen este tipo de prueba durante sus consultas telefónicas.

\section{Puntaje de Roth para evaluación de la disnea}

El puntaje de Roth $^{2}$ es una herramienta de uso sencillo que fue validada en voluntarios sanos y pacientes hospitalizados a través de un estudio que usó como prueba de referencia la oximetría de pulso. Sin embargo, este instrumento no fue validado en el ámbito de la atención primaria. Consiste en solicitarle al paciente que inspire profundamente y cuente en voz alta del 1 al 30 , en su propio idioma. La persona que realiza la evaluación debe medir el tiempo en segundos durante el cual el paciente logra mantener el conteo sin volver a inspirar.

Si el paciente logra mantener el conteo durante ocho segundos o menos, existe alta probabilidad de que su saturación de oxígeno evaluada a través de una oximetría de pulso sea menor a $95 \%$ (sensibilidad: $78 \%$; especificidad: $71 \%$ ). Usando como punto de corte los cinco segundos, la sensibilidad de esta prueba del $91 \%$.
Sin embargo, sólo seis de los 50 expertos consultados había usado alguna vez el puntaje de Roth, y la mayoría ni siquiera lo conocía. Por otro lado, este grupo consultado manifestó la preocupación de que el uso masivo de este puntaje en el contexto de la epidemia por Covid-19 podría causar más daños que beneficios, ya que que esta herramienta no sería lo suficientemente sensible como para identificar enfermedad grave y podría conducir a brindar un falso reaseguro.

\section{Dispositivos de oximetría suministrados a paciente y} aplicaciones para teléfonos inteligentes

Si bien es común el uso de dispositivos de oximetría por pacientes con enfermedades respiratorias crónicas, no se ha evaluado su uso en el entorno de la atención primaria.

Respecto de las aplicaciones para los teléfonos inteligentes, existe muy poca investigación publicada al respecto. Un estudio pequeño, que incluyó pacientes hospitalizados, informó una correlación alta (mayor de $98 \%$ ) entre la lectura del teléfono inteligente y un oxímetro de pulso. Sin embargo, dada la diversidad de dispositivos y aplicaciones, su implementación en la práctica clínica debe interpretarse con precaución y solamente utilizarse en el contexto de una evaluación clínica más amplia.

\section{Resumen de las opiniones del grupo de expertos para} la evaluación a distancia de la disnea y el compromiso respiratorio

Dada la escasa evidencia disponible para la toma de decisiones en el contexto de la actual pandemia, el 20/3/2020 Greenhalgh y col. decidieron realizar una encuesta a una muestra de 50 médicos británicos que evalúan regularmente pacientes por teléfono.

Esta encuesta arrojó como resultado una serie de recomendaciones que pueden ser resumidas en una lista de siete preguntas:

1. Pídale al paciente que describa el problema de su respiración en sus propias palabras y evalúe la facilidad y la comodidad de su discurso. Haga preguntas abiertas y escuche si el paciente puede completar sus oraciones. Por ejemplo:

¿Cómo está tu respiración hoy?

2. Realice las siguientes tres preguntas (son las que usa el sistema británico de emergencias y no han sido evaluadas a través de una investigación formal):

¿Su falta de aire es tal que no puede hablar más que unas pocas palabras?

¿Está respirando con más fuerza o más rápidamente que lo habitual, cuando no está realizando actividades?

¿Se siente tan mal que no puede hacer las cosas que hace habitualmente?

3. Focalice su evaluación en el proceso y los cambios que ha sentido el paciente por sobre su situación actual (priorice la "película" por sobre la "foto") ya que una historia de deterioro rápido es más importante que la magnitud de la disnea que el paciente siente actualmente. Pregunte por ejemplo: 
¿Qué podía usted hacer ayer (o hace unos días) que no puede hacer hoy?

¿Qué sucede hoy que ayer no ocurría, que lo deja sin aire?

¿Qué actividad tiene que realizar hoy para que le falte el aire?

4. Interprete la disnea en el contexto de la historia de los signos físicos del paciente que puedan ser evaluados a distancia. Por ejemplo, sibilancias audibles por el propio paciente o por alguna persona conviviente y/o un informe verbal de cianosis peri-oral son banderas rojas para quien está realizando la evaluación.

Descargo de responsabilidad de los autores: Este artículo de revisión no ha sido revisado por pares. No debe reemplazar el juicio clínico individual y se deben verificar las fuentes citadas. Las opiniones expresadas en este comentario representan las opiniones de los autores y no necesariamente las de la institución anfitriona, el Sistema Nacional de Salud de UK, el NIHR o el Departamento de Salud y Atención Social de Gran Bretaña.

Las opiniones no son un sustituto del consejo médico profesional.

\section{Comentario}

Estamos viviendo la pandemia del COVID-19 y eso nos sitúa en un escenario nuevo e incierto. Con la finalidad de disminuir los contactos presenciales con nuestros pacientes, quienes nos desempeñamos en el ámbito de la atención primaria nos vemos obligados a enfrentarnos a nuevos desafíos y modalidades de atención, a través de dispositivos celulares o telemedicina. Entendemos que la premura a las que nos obliga actuar la actual emergencia sanitaria impulsó a estos autores, de reconocida trayectoria en la medicina basada en la evidencia, a publicar una revisión no sistemática de formato puramente narrativo, cuyas principales conclusiones se basan en una encuesta realizada muy velozmente a un grupo de 50 expertos, de la que no han explicitado los criterios de selección de los participantes, ni la sistemática de su diseño.

Con las limitaciones metodológicas que ellos mismos reconocen, nos informan que las herramientas disponibles para la evaluación de la disnea mediante dispositivos a distancia no fueron evaluadas en el ámbito de la atención ambulatoria y nos recomiendan usar nuestra experiencia clínica para la toma de decisiones en el contexto de dichas consultas. También hacen una advertencia con una mirada de prevención cuaternaria sobre los posibles riesgos de usar tecnologías no evaluadas previamente en el ámbito en cuestión, a pesar que nuestro sentido común nos haga pensar que pudieran resultar de utilidad. Esta última mirada es para mí el aspecto que más valoro de este tipo de comunicación.

\section{Conclusiones del comentador}

Aún en el contexto de la urgencia y las necesidades que nos genera esta pandemia, debemos ser reflexivos y respetar nuestros principios básicos de atención, basando nuestras decisiones en la mejor evidencia disponible, que en este momento parece provenir de una recomendación de expertos que ha incluido una mirada de prevención cuaternaria.

Jinich P. Herramientas para la evaluación de la disnea y el compromiso respiratorio en pacientes atendidos por teléfono o video-llamada en el contexto de la pandemia por COVID-19. Evid Actual Pract Ambul. 2020;23(2):e002055. Comentado de: Greenhalgh T et al. Are there any evidence-based ways of assessing dyspnoea (breathlessness) by telephone or video. 02/04/2020. The Center for Evidence-Based Medicine. University of Oxford. Disponible en URL: https://www.cebm.net/covid-19/are-there-any-evidence-based-ways-of-assessing-dyspnoea-breathlessness-by-telephone-or-video/ (último acceso $13 / 04 / 2020)$

\section{Referencias}

1. Greenhalgh T, Kotze K, Van-Der-Westhuizen HM. Are there any evidence-based ways of assessing dyspnoea (breathlessness) by telephone or video; 2020. Available from: https://www.cebm.net/covid-19/are-there-any-evidence-based-ways-of-assessing-dyspnoea-breathlessness-by-telephoneor-video/ [Last access: 2020-04-13]

2. Chorin E, Padegimas A, Havakuk O. Assessment of Respiratory Distress by the RothScore. Clin Cardiol. 2016;39(11):636-639. Available from: $10.1002 /$ clc. 22586 . 